\title{
Dynamic Model of Decentralized Systems with Informational Connection
}

\author{
Yasutaka Fujimoto, Member, IEEE, Takahiro Yakoh, Member, IEEE, and \\ Kouhei Ohnishi, Fellow, IEEE
}

\begin{abstract}
This paper presents a design method of decentralized systems with informational connection. The informational connection in this paper denotes a event link which establish a signal links among physical controlled plants via communication network. A dynamic transition of informational connection among decentralized systems is considered and the mathematical structures are discussed using a concept of eigenvalues and eigen-connections over the Galois field $G F(2)$. The global system has variable structure characteristics due to the transition of informational connection. Examples of decentralized variable structure systems are shown. In an industry field, there are many engineering systems that have dynamic transition of informational connection. The mathematical model would be useful for analysis and synthesis of various informationally connected systems.
\end{abstract}

\section{INTRODUCTION}

$\mathrm{R}$ ECENT years, many industrial systems are becoming too complex to understand how they work. Since these systems are designed by hierarchical and distributed methods, it is difficult to guarantee the stability and the safety of the global systems. The difficulty comes from an unexpected interaction among independently designed subsystems. There are several works related to distributed systems[1]-[6].

Fig. 1 shows a general concept of hierarchical distributed systems that has a event link layer and a signal link layer. The signal links among physical controlled plants are established via communication network due to event links among connection controllers. In general, many industrial systems are implicitly designed so that the information flow will change due to conditions of the physical plant and the controller. Namely, the information flow is determined by their logical variables of the conditions. This double-layer model has the potential advantage of clearness, performance, and reuse effectiveness of the modules. Explicitly considering the double-layer concept, the model is expected to settle the difficulties of large complex systems. Similar concepts are found in Responsive Processor[7] and IEC61499 Function Blocks[8]. They, however, just provide frame works.

In this paper, analysis and design method for the logic layer of the information flow is proposed because it is important to systematically handle transitions of information flow depending on the logical condition variable. A dy-

Manuscript received November 14, 2000; revised February 15, 2002. Y. Fujimoto is with the Department of Electrical and Computer Engineering, Yokohama National University, Tokiwadai, Yokohama 240-8501 Japan. T. Yakoh and K. Ohnishi are with the Department of System Design Engineering, Keio University, Hiyoshi, Yokohama 223-8522 Japan. E-mail: fujimoto@ynu.ac.jp, yakoh@sd.keio.ac.jp, ohnishi@sd.keio.ac.jp namic transition of informational connection is considered and the mathematical structure is discussed using a concept of eigenvalues and eigen-connections over the Galois field $G F(2)$. The global system has variable structure characteristics due to the transition of informational connection.

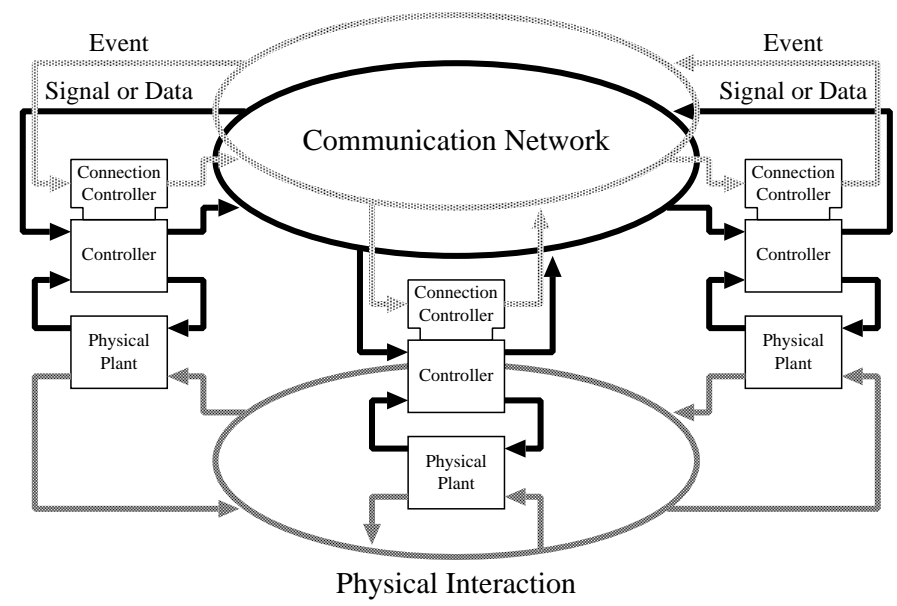

Fig. 1. Basic concept of informationally connected systems

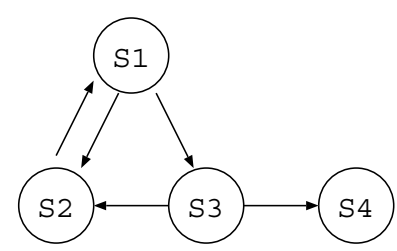

Fig. 2. An example of an informationally connected system

\section{Dynamic Model of Connection Systems}

Consider informationally connected systems which consist of $n$ sub-systems. A connection matrix $X \in G F(2)^{n \times n}$ is defined as:

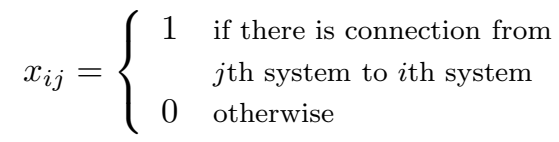

where $x_{i j}$ is the $(i, j)$ element of the matrix $X . G F(2)$ represents the Galois field having two elements $\{0,1\}$. Fig. 1 shows an example of an informationally connected system 
which consists of sub-systems $\left\{S_{1}, S_{2}, S_{3}, S_{4}\right\}$. The connection matrix of this system becomes:

$$
X=\begin{gathered}
S_{1 D} S_{2 D} S_{3 D} S_{4 D} \\
S_{1 R} \\
S_{2 R} \\
S_{3 R} \\
S_{4 R}
\end{gathered}\left[\begin{array}{llll}
0 & 1 & 0 & 0 \\
1 & 0 & 1 & 0 \\
1 & 0 & 0 & 0 \\
0 & 0 & 1 & 0
\end{array}\right]
$$

where the subscript $D$ and $R$ represent Donator and $R e$ ceptor of information[2].

The operations on the Galois field $G F(2)$ are defined as follows $[9]$ :

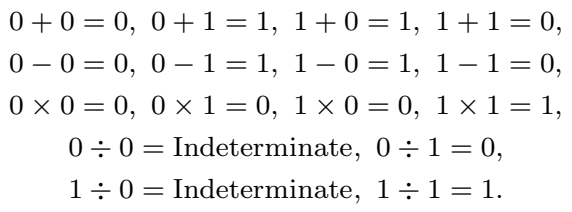

In this paper, a vector space in a set $G F(2)^{n \times n}$ is called $a$ connection space. A norm of a connection $X \in G F(2)^{n \times n}$ is defined as $G F(2)^{n \times n} \rightarrow N$ by $|X|=\sum_{i=1}^{n} \sum_{j=1}^{n} X_{i j}$, where $N$ denotes a set of all natural numbers. Note that the addition in the summation takes on $N$. A distance between two connected systems $X_{1}$ and $X_{2}$ is expressed as $\left|X_{1}-X_{2}\right|$. Distance represents how different two informationally connected systems are. An inner product of two connections $X, Y \in G F(2)^{n \times n}$ is defined as $G F(2)^{n \times n} \times G F(2)^{n \times n} \rightarrow$ $N$ by $\langle X, Y\rangle=\sum_{i=1}^{n} \sum_{j=1}^{n} X_{i j} Y_{i j}$. The connection space becomes metric space by these definitions. The metric space is needed to express a dynamic transition of an informational connection because a fluctuation is based on a concept of a distance.

Conventionally, a concept of a distance was not considered in a connection analysis because it uses a Boolean algebra. Boolean algebra is applicable not to a dynamic transition analysis but to a kinematics structure analysis.

Assume that a transition of an informational connection is affected by a current state of the connection and environmental event inputs, then we have informationally connected systems of the general form

$$
X(t+1)=f\left(X(t), u_{1}(t), u_{2}(t), \ldots, u_{m}(t), t\right)
$$

where $t$ is a unit time and $u_{i}$ is event input from environment and controller. Each element of the connection matrix $X$ represents not only the connection itself but also the condition of the transition of the information flow structure. Also event input $u_{i}$ represents the condition of the transition depending on status of the low layer controller and the physical plant.

\section{A. Dynamic Model affected by Donator Connection}

As a sub-class of the system (3), we have a linear dynamic model of informational connection affected by a current state of the Donator connection.

$$
X(t+1)=A_{D}(t) X(t)+b_{D}(t) u_{D}(t)^{T}
$$

where $A_{D}(t) \in G F(2)^{n \times n}$ represents Donator matrix, $u_{D}(t) \in G F(2)^{n}$ represents a external event input to change Donator connection, and $b_{D}(t) \in G F(2)^{n}$ represents its coefficient matrix. Note that $i$ th column of the connection matrix $X(t)$ represents Donator connection of $i$ th subsystem. The multiplication of $X(t)$ by $A_{D}(t)$ from the left affects each column of $X(t)$, i. e., Donator connection of each subsystem.

\section{Example $I I-1$}

Consider the Donator-type informationally connected system

$$
\left\{A_{D}, b_{D}, X(0)\right\}=\left\{\left[\begin{array}{lll}
0 & 1 & 0 \\
0 & 0 & 1 \\
1 & 0 & 0
\end{array}\right],\left[\begin{array}{l}
0 \\
0 \\
1
\end{array}\right],\left[\begin{array}{lll}
0 & 0 & 0 \\
0 & 0 & 0 \\
0 & 0 & 0
\end{array}\right]\right\}
$$

and the external event input $u_{D}=\left[u_{D 1}, u_{D 2}, u_{D 3}\right]^{T}$. When an impulsive event input is imposed on the first subsystem,

$$
u_{D 1}(t)= \begin{cases}1 & \text { if } t=0 \\ 0 & \text { otherwise }\end{cases}
$$

the impulse response of the transition of the connection becomes as shown in Fig. 3 (a). In this case, a periodic transition can be observed.

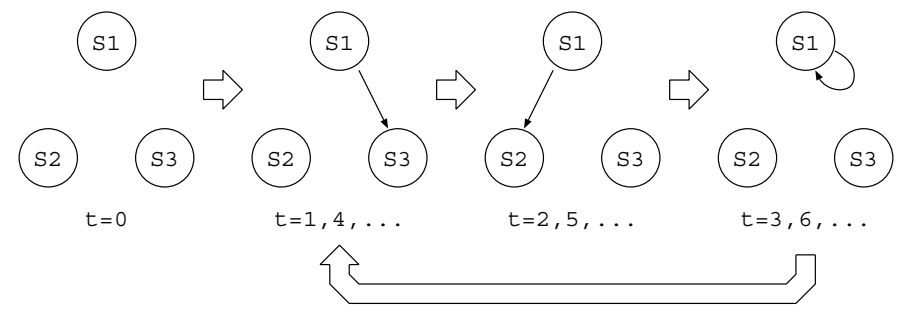

(a) Donator-type connection system

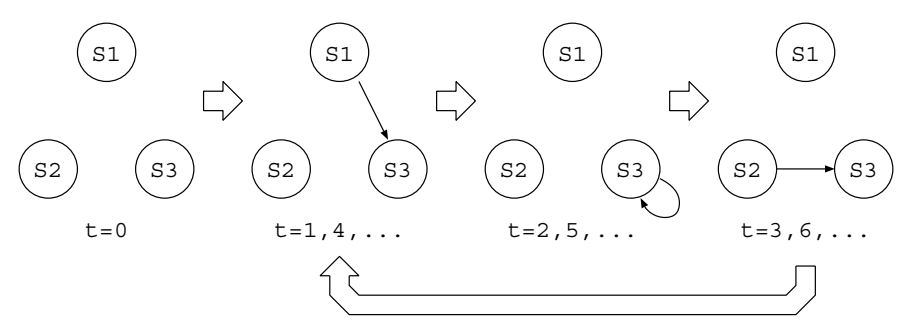

(b) Receptor-type connection system

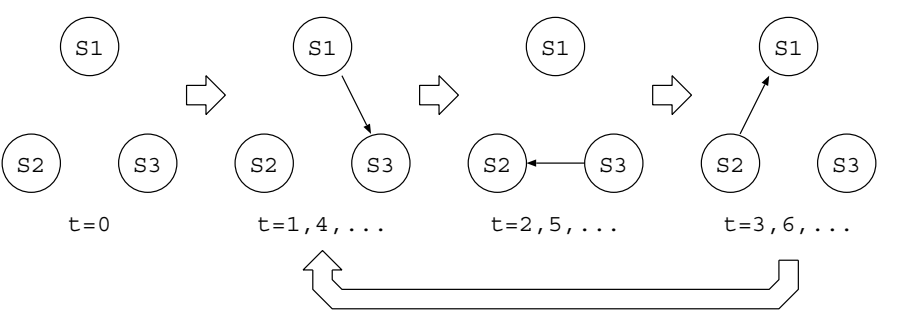

(c) Donator-receptor-type connection system

Fig. 3. Impulse transition of connection system

\section{B. Dynamic Model affected by Receptor Connection}

As a dual system of the donator model (4), we have a linear dynamic model of informational connection affected 
by the Receptor connection

$$
X(t+1)=X(t) A_{R}(t)^{T}+u_{R}(t) b_{R}(t)^{T}
$$

where $A_{R}(t) \in G F(2)^{n \times n}$ represents Receptor matrix, $u_{R}(t) \in G F(2)^{n}$ represents a external event input to change Receptor connection, and $b_{R}(t) \in G F(2)^{n}$ represents its coefficient matrix. Since $i$ th row of the connection matrix $X(t)$ represents Receptor connection of $i$ th subsystem, the multiplication of $X(t)$ by $A_{R}(t)$ from the right affects each row of $X(t)$, i. e., Receptor connection of each subsystem.

\section{Example II-2}

Consider the Receptor-type informationally connected system

$$
\left\{A_{R}, b_{R}, X(0)\right\}=\left\{\left[\begin{array}{lll}
0 & 1 & 0 \\
0 & 0 & 1 \\
1 & 0 & 0
\end{array}\right],\left[\begin{array}{l}
0 \\
0 \\
1
\end{array}\right],\left[\begin{array}{lll}
0 & 0 & 0 \\
0 & 0 & 0 \\
0 & 0 & 0
\end{array}\right]\right\}
$$

and the external event input $u_{R}=\left[u_{R 1}, u_{R 2}, u_{R 3}\right]^{T}$. When an impulsive event input is imposed on the first subsystem, the impulse response of the transition of the connection becomes as shown in Fig. 3 (b). Also in this case, a periodic transition can be observed.

\section{Dynamic Model affected by Donator and Receptor Con- nection}

In General, both Donator and Receptor connection have influence on the next state of the connection. A Combination of (4) and (7) yields the general linear dynamic model:

$$
\begin{aligned}
& X(t+1)=A_{D}(t) X(t) A_{R}(t)^{T}+b_{D}(t) u(t) b_{R}(t)^{T} \\
& +b_{D}(t) u_{D}(t)^{T} A_{R}(t)^{T}+A_{D}(t) u_{R}(t) b_{R}(t)^{T}
\end{aligned}
$$

where $X(t), A_{D}(t), A_{R}(t) \in G F(2)^{n \times n}, \quad b_{D}(t), b_{R}(t) \in$ $G F(2)^{n} \cdot u(t) \in G F(2)$ represents a external event input to change the connection directly and $u_{D}(t), u_{R}(t) \in G F(2)^{n}$ represent external event inputs to change Donator and Receptor connection, respectively. The model can express transmission of information from a subsystem to others. This is one representation of decentralized system.

\section{Example II-3}

Consider the Donator-Receptor-type informationally connected system

$$
\begin{aligned}
& \left\{A_{D}, A_{R}, b_{D}, b_{R}, X(0)\right\}= \\
& \left\{\left[\begin{array}{lll}
0 & 1 & 0 \\
0 & 0 & 1 \\
1 & 0 & 0
\end{array}\right],\left[\begin{array}{lll}
0 & 1 & 0 \\
0 & 0 & 1 \\
1 & 0 & 0
\end{array}\right],\left[\begin{array}{l}
0 \\
1 \\
0
\end{array}\right],\left[\begin{array}{l}
0 \\
0 \\
1
\end{array}\right],\left[\begin{array}{lll}
0 & 0 & 0 \\
0 & 0 & 0 \\
0 & 0 & 0
\end{array}\right]\right\} .
\end{aligned}
$$

The impulse response from the event input $u(t)$ becomes as shown in Fig. 3 (c).

\section{Structure of Connection Systems}

In this section, the mathematical structure of the connection space is discussed based on the concept of eigenvalue and eigen-connection.

\section{A. Eigenvalues}

It is well known that eigenvalues $\lambda \in G F\left(2^{\ell}\right)$ and eigenvectors $v \in G F\left(2^{\ell}\right)^{n}$ exist for any matrix $A \in G F(2)^{n \times n}$ which satisfy $\lambda v=A v . \quad \ell$ is an adequate positive integer mentioned below. Then eigenvalues $\lambda$ can be obtained by solving a characteristic equation $\psi(\lambda)=\operatorname{det}(\lambda I-A)=0$. The equation $\psi(\lambda)=0$ is solvable when we consider an extension field of $G F(2)$.

Let characteristic polynomials of Donator matrix $A_{D}$ and Receptor matrix $A_{R}$ be given by

$$
\begin{aligned}
\psi_{D}(\lambda) & =\operatorname{det}\left(\lambda I-A_{D}\right) \\
& =\lambda^{n}+a_{D n} \lambda^{n-1}+\ldots+a_{D 2} \lambda+a_{D 1} \\
\psi_{R}(\lambda) & =\operatorname{det}\left(\lambda I-A_{R}\right) \\
& =\lambda^{n}+a_{R n} \lambda^{n-1}+\ldots+a_{R 2} \lambda+a_{R 1} .
\end{aligned}
$$

where $a_{D i}, a_{R i} \in G F(2)$ for $1 \leq i \leq n$. The characteristics polynomials $\psi_{D}(\lambda)$ and $\psi_{R}(\lambda)$ are elements in a set $G F(2)[\lambda]$ which is the ring of univariate polynomials over $G F(2)$. The factorizations of (11) and (12) over $G F(2)[\lambda]$ are represented by

$$
\begin{aligned}
& \psi_{D}(\lambda)=\psi_{D 1}(\lambda)^{n_{1}} \psi_{D 2}(\lambda)^{n_{2}} \cdots \psi_{D p_{D}}(\lambda)^{n_{p_{D}}} \\
& \psi_{R}(\lambda)=\psi_{R 1}(\lambda)^{n_{1}} \psi_{R 2}(\lambda)^{n_{2}} \cdots \psi_{R p_{R}}(\lambda)^{n_{p_{R}}}
\end{aligned}
$$

where $\psi_{D i}(\lambda)$ and $\psi_{R j}(\lambda)$ are irreducible polynomials over $G F(2)[\lambda]$.

Let the degrees of irreducible polynomials $\psi_{D i}(\lambda)$ and $\psi_{R j}(\lambda)$ be $\ell_{D i}$ and $\ell_{R i}$ for $1 \leq i \leq p_{D}$ and $1 \leq j \leq p_{R}$, respectively. Then characteristic polynomials $\psi_{D}(\lambda)$ and $\psi_{R}(\lambda)$ can be factorized into the form

$$
\begin{aligned}
& \psi_{D}(\lambda)=\left(\lambda-\lambda_{D 1}\right)^{n_{1}}\left(\lambda-\lambda_{D 2}\right)^{n_{2}} \cdots\left(\lambda-\lambda_{D_{p_{D}}}\right)^{n_{p_{D}}} \\
& \psi_{R}(\lambda)=\left(\lambda-\lambda_{R 1}\right)^{n_{1}}\left(\lambda-\lambda_{R 2}\right)^{n_{2}} \cdots\left(\lambda-\lambda_{R_{p_{R}}}\right)^{n_{p_{R}}}
\end{aligned}
$$

over an extension field $G F\left(2^{\ell}\right)$, where

$$
\ell=\operatorname{LCM}\left(\ell_{D 1}, \ldots, \ell_{D p_{D}}, \ell_{R 1}, \ldots, \ell_{R p_{R}}\right)
$$

$\lambda_{D i}, \lambda_{R j} \in G F\left(2^{\ell}\right)$ correspond to eigenvalues of $A_{D}$ and $A_{R}$. In this paper, they are named Donator eigenvalue and Receptor eigenvalue.

\section{Example III-1}

For the system $\left\{A_{D}, A_{R}, b_{D}, b_{R}, X(0)\right\}$ of Example II-3, the characteristic polynomial of $A_{D}$ and $A_{R}$ can be factorized into irreducible polynomials:

$$
\psi_{D}(\lambda)=\psi_{R}(\lambda)=\lambda^{3}+1=(\lambda+1)\left(\lambda^{2}+\lambda+1\right) .
$$

Since the degrees of irreducible polynomials are $\ell_{D 1}=$ $\ell_{R 1}=1, \ell_{D 2}=\ell_{R 2}=2$, the order of the extension field is obtained from (17) as $\ell=\operatorname{LCM}(1,2,1,2)=2$. Let the root of one of second degree irreducible polynomial $\lambda^{2}+\lambda+1=0$ be $\alpha$. (18) is further factorized on polynomial modulo $\alpha^{2}+\alpha+1$.

$$
\psi_{D}(\lambda)=\psi_{R}(\lambda)=(\lambda+1)(\lambda+\alpha)\left(\lambda+\alpha^{2}\right) .
$$


Therefore the eigenvalues of $A_{D}$ and $A_{R}$ are $\lambda_{D 1}=\lambda_{R 1}=$ $1, \lambda_{D 2}=\lambda_{R 2}=\alpha, \lambda_{D 3}=\lambda_{R 3}=\alpha^{2}$.

Fig. 4 illustrates the pole plot of (19). We shall call this pole plot GF-plane for convenience. In GF-plane, the origin denotes the element of zero. The points on the unit circle denote all elements of $G F\left(2^{\ell}\right)$ except 0 . In this case, the pole $\alpha$ operates a state of connection to rotate by $2 \pi / 3$ [rad] on GF-plane. Also the operation of $\alpha^{2}$ is by $4 \pi / 3$ [rad]. Therefore, we can see the period of this system is three.

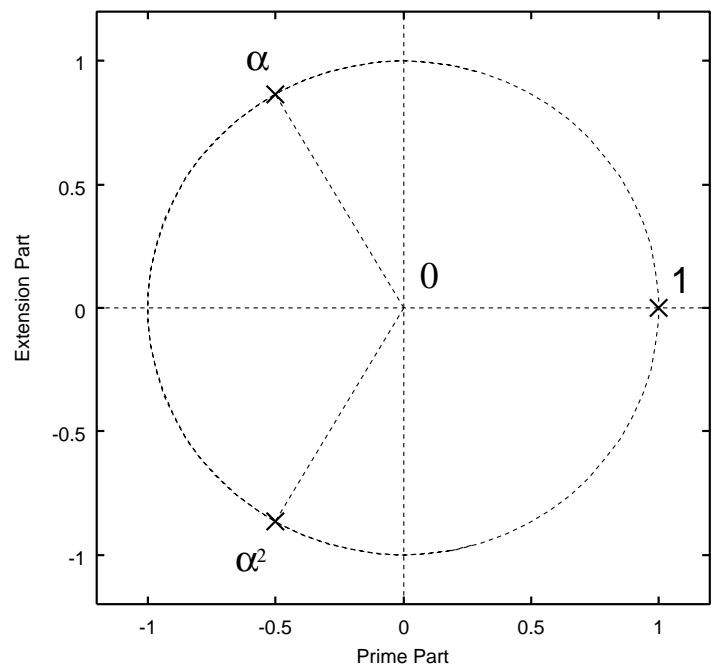

Fig. 4. Pole plot in $G F$-plane

\section{B. Eigen-connections}

Let $i$ th generalized eigenvector of $A_{D}$ be $v_{D i}, j$ th generalized eigenvector of $A_{R}$ be $v_{R j}$. One of eigen-connections $W_{i j}$ is defined by

$$
W_{i j}=v_{D i} v_{R j}^{T} .
$$

If there is no duplicate eigenvalues in each of $A_{D}$ and $A_{R}$, the eigen-connection satisfies

$$
\lambda_{i j} W_{i j}=A_{D} W_{i j} A_{R}^{T}
$$

where $\lambda_{i j}=\lambda_{D i} \lambda_{R j}$ corresponds to the eigenvalue of connection systems. The eigen-connections are special connections whose shapes are invariant during dynamic transition. The norms are, however, variant due to the corresponding eigenvalues.

Since the number of eigen-connections in a connection system is $n^{2}$ and they are independent each other, any connection $X$ can be expressed by the sum of them

$$
X=\sum_{i=1}^{n} \sum_{j=1}^{n} a_{i j} W_{i j}
$$

where $a_{i j} \in G F\left(2^{\ell}\right)$. The eigen-connections are basis which span the connection space.

\section{Example III-2}

For the system $\left\{A_{D}, A_{R}, b_{D}, b_{R}, X(0)\right\}$ of Example III-A1 , the eigenvectors of $A_{D}$ and $A_{R}$ are given by $v_{D 1}=v_{R 1}=$ $[1,1,1]^{T}, v_{D 2}=v_{R 2}=\left[1, \alpha, \alpha^{2}\right]^{T}, v_{D 3}=v_{R 3}=\left[1, \alpha^{2}, \alpha\right]^{T}$. From (20), the eigen-connections are given by

$$
\begin{aligned}
\left\{W_{11},\right. & \left.W_{12}, W_{13}, W_{21}, W_{22}, W_{23}, W_{31}, W_{32}, W_{33}\right\} \\
= & \left\{\left[\begin{array}{lll}
1 & 1 & 1 \\
1 & 1 & 1 \\
1 & 1 & 1
\end{array}\right],\left[\begin{array}{lll}
1 & \alpha & \alpha^{2} \\
1 & \alpha & \alpha^{2} \\
1 & \alpha & \alpha^{2}
\end{array}\right],\left[\begin{array}{lll}
1 & \alpha^{2} & \alpha \\
1 & \alpha^{2} & \alpha \\
1 & \alpha^{2} & \alpha
\end{array}\right],\right. \\
& {\left[\begin{array}{lll}
1 & 1 & 1 \\
\alpha & \alpha & \alpha \\
\alpha^{2} & \alpha^{2} & \alpha^{2}
\end{array}\right],\left[\begin{array}{lll}
1 & \alpha & \alpha^{2} \\
\alpha & \alpha^{2} & 1 \\
\alpha^{2} & 1 & \alpha
\end{array}\right],\left[\begin{array}{lll}
1 & \alpha^{2} & \alpha \\
\alpha & 1 & \alpha^{2} \\
\alpha^{2} & \alpha & 1
\end{array}\right], } \\
& {\left.\left[\begin{array}{lll}
1 & 1 & 1 \\
\alpha^{2} & \alpha^{2} & \alpha^{2} \\
\alpha & \alpha & \alpha
\end{array}\right],\left[\begin{array}{lll}
1 & \alpha & \alpha^{2} \\
\alpha^{2} & 1 & \alpha \\
\alpha & \alpha^{2} & 1
\end{array}\right],\left[\begin{array}{lll}
1 & \alpha^{2} & \alpha \\
\alpha^{2} & \alpha & 1 \\
\alpha & 1 & \alpha^{2}
\end{array}\right]\right\} . }
\end{aligned}
$$

Then their eigenvalues are given by

$$
\begin{gathered}
\left\{\lambda_{11}, \lambda_{12}, \lambda_{13}, \lambda_{21}, \lambda_{22}, \lambda_{23}, \lambda_{31}, \lambda_{32}, \lambda_{33}\right\} \\
=\left\{\begin{array}{lll}
1, & \alpha, & \alpha^{2}, \\
\alpha, & \alpha^{2}, & 1 \\
\alpha^{2}, & 1, & \alpha
\end{array}\right\} .
\end{gathered}
$$

There are three steady eigen-connections $W_{11}, W_{23}, W_{32}$ and other six periodic eigen-connections.

\section{Conjugate Connections}

The connection space representation using eigenconnections includes element $\alpha$ of the extension field $G F\left(2^{\ell}\right)$. In this section, another basis of connection space excluding $\alpha$ is introduced using sets of conjugate connections.

Let one of the eigenvalues be $\beta$. Then a set of the eigenvalues $\left\{\beta, \beta^{2}, \beta^{4}, \ldots, \beta^{2^{d-1}}\right\}$ exist and they become conjugate, where $d$ is a minimum positive integer satisfying $\beta^{2^{d}}=\beta$. For example, two eigenvalues $\alpha, \alpha^{2}$ in Example III- $A-1$ are conjugate. We can examine it by $(\lambda+\alpha)\left(\lambda+\alpha^{2}\right)=\lambda^{2}+\lambda+1$.

Let $i$ th set of conjugate eigenvalues of $A_{D}$ be $\left\{\beta_{i}, \beta_{i}^{2}, \beta_{i}^{4}, \ldots, \beta_{i}^{2^{d_{i}-1}}\right\}$ and their eigenvectors be $\left\{v_{\beta_{i} 1}, v_{\beta_{i} 2}\right.$ $\left., \ldots, v_{\beta_{i} d_{i}}\right\}$. Consider $n \times n$ matrix $G_{D} \in G F\left(2^{\ell}\right)$

$$
G_{D}=\text { block } \operatorname{diag}\left(G_{D 1}, G_{D 2}, \ldots, G_{D i}, \ldots\right)
$$

where

$$
G_{D i}=\left[\begin{array}{ccccc}
1 & 1 & 1 & \cdots & 1 \\
\beta_{i} & \beta_{i}^{2} & \beta_{i}^{4} & & \beta_{i}^{2^{d_{i}-1}} \\
\beta_{i}^{2} & \beta_{i}^{4} & \beta_{i}^{8} & & \beta_{i}^{2^{d_{i}}} \\
\vdots & & & & \vdots \\
\beta_{i}^{d_{i}-1} & \beta_{i}^{2\left(d_{i}-1\right)} & \beta_{i}^{4\left(d_{i}-1\right)} & \cdots & \beta_{i}^{2^{d_{i}-1}\left(d_{i}-1\right)}
\end{array}\right] .
$$

Then let the inverse matrix of $G_{D}$ be

$$
H_{D}=G_{D}{ }^{-1}=\operatorname{block} \operatorname{diag}\left(H_{D 1}, H_{D 2}, \ldots, H_{D i}, \ldots\right)
$$

where

$$
H_{D i}=G_{D i}{ }^{-1}=\left[\begin{array}{cccc}
h_{D i 11} & h_{D i 12} & \cdots & h_{D i 1 d} \\
h_{D i 21} & h_{D i 22} & & h_{D i 2 d} \\
\vdots & & & \vdots \\
h_{D i d 1} & h_{D i d 2} & \cdots & h_{D i d d}
\end{array}\right]
$$


Let us consider new vectors $v_{\beta_{i} j}^{\prime}$ defined by $v_{\beta_{i} j}^{\prime}=$ $\sum_{k=1}^{d_{i}} h_{D i k j} v_{\beta_{i} k}$ i. e.

$$
V_{D}^{\prime}=V_{D} H_{D}
$$

where

$$
\begin{aligned}
V_{D} & =\left[v_{\beta_{1} 1}, v_{\beta_{1} 2}, \ldots, v_{\beta_{1} d_{1}}, v_{\beta_{2} 1}, v_{\beta_{2} 2}, \ldots, v_{\beta_{2} d_{2}}, \ldots,\right] \\
V_{D}^{\prime} & =\left[v_{\beta_{1} 1}^{\prime}, v_{\beta_{1} 2}^{\prime}, \ldots, v_{\beta_{1} d_{1}}^{\prime}, v_{\beta_{2} 1}^{\prime}, v_{\beta_{2} 2}^{\prime}, \ldots, v_{\beta_{2} d_{2}}^{\prime}, \ldots,\right]
\end{aligned}
$$

In a similar manner, let $j$ th set of conjugate eigenvalues of $A_{R}$ be $\left\{\gamma_{j}, \gamma_{j}^{2}, \gamma_{j}^{4}, \ldots, \gamma_{j}^{2^{r_{j}-1}}\right\}$ and their eigenvectors be $v_{\gamma_{j} 1}, v_{\gamma_{j}}, \ldots, v_{\gamma_{j} r_{j}}$. Then we compute

$$
V_{R}^{\prime}=V_{R} H_{R}
$$

where

$$
\begin{aligned}
V_{R} & =\left[v_{\gamma_{1} 1}, v_{\gamma_{1} 2}, \ldots, v_{\gamma_{1} r_{1}}, v_{\gamma_{2} 1}, v_{\gamma_{2} 2}, \ldots, v_{\gamma_{2} r_{2}}, \ldots,\right] \\
V_{R}^{\prime} & =\left[v_{\gamma_{1} 1}^{\prime}, v_{\gamma_{1} 2}^{\prime}, \ldots, v_{\gamma_{1} r_{1}}^{\prime}, v_{\gamma_{2} 1}^{\prime}, v_{\gamma_{2} 2}^{\prime}, \ldots, v_{\gamma_{2} r_{2}}^{\prime}, \ldots,\right]
\end{aligned}
$$

The conjugate basis of connection space is given by

$$
W_{i j}^{\prime}=v_{D i}^{\prime} v_{R j}^{\prime T}
$$

where $v_{D i}^{\prime}$ denotes $i$ th column vector of $V_{D}^{\prime}$ and $v_{R j}^{\prime}$ denotes $j$ th column vector of $V_{R}^{\prime}$.

\section{Example III-3}

For the system of Example III-A-1, the conjugate connections are computed as follows. We compute

$$
G_{D}=G_{R}=\left[\begin{array}{ccc}
1 & 0 & 0 \\
0 & 1 & 1 \\
0 & \alpha & \alpha^{2}
\end{array}\right]
$$

Then $H_{D}=G_{D}^{-1}$ and $H_{R}=G_{R}^{-1}$ are given by

$$
H_{D}=H_{R}=\left[\begin{array}{ccc}
1 & 0 & 0 \\
0 & \alpha^{2} & 1 \\
0 & \alpha & 1
\end{array}\right]
$$

From (29) and (30)

$$
\left[v_{D 1}^{\prime}, v_{D 2}^{\prime}, v_{D 3}^{\prime}\right]=\left[v_{R 1}^{\prime}, v_{R 2}^{\prime}, v_{R 3}^{\prime}\right]=\left[\begin{array}{lll}
1 & 1 & 0 \\
1 & 0 & 1 \\
1 & 1 & 1
\end{array}\right] .
$$

Therefore, the conjugate basis of connection space is given by

$$
\begin{aligned}
& \left\{W_{11}^{\prime}, W_{12}^{\prime}, W_{13}^{\prime}, W_{21}^{\prime}, W_{22}^{\prime}, W_{23}^{\prime}, W_{31}^{\prime}, W_{32}^{\prime}, W_{33}^{\prime}\right\} \\
& =\left\{\left[\begin{array}{lll}
1 & 1 & 1 \\
1 & 1 & 1 \\
1 & 1 & 1
\end{array}\right],\left[\begin{array}{lll}
1 & 0 & 1 \\
1 & 0 & 1 \\
1 & 0 & 1
\end{array}\right],\left[\begin{array}{lll}
0 & 1 & 1 \\
0 & 1 & 1 \\
0 & 1 & 1
\end{array}\right],\right. \\
& {\left[\begin{array}{lll}
1 & 1 & 1 \\
0 & 0 & 0 \\
1 & 1 & 1
\end{array}\right],\left[\begin{array}{lll}
1 & 0 & 1 \\
0 & 0 & 0 \\
1 & 0 & 1
\end{array}\right],\left[\begin{array}{lll}
0 & 1 & 1 \\
0 & 0 & 0 \\
0 & 1 & 1
\end{array}\right],} \\
& \left.\left[\begin{array}{lll}
0 & 0 & 0 \\
1 & 1 & 1 \\
1 & 1 & 1
\end{array}\right],\left[\begin{array}{lll}
0 & 0 & 0 \\
1 & 0 & 1 \\
1 & 0 & 1
\end{array}\right],\left[\begin{array}{lll}
0 & 0 & 0 \\
0 & 1 & 1 \\
0 & 1 & 1
\end{array}\right]\right\} \text {. }
\end{aligned}
$$

\section{Autonomous Rhythm of Connections}

In this section, the period of transition of connection systems is discussed. For $i$ th eigenvalue $\lambda_{D i}$ of $A_{D}$, the period $p_{D i}$ is defined by the minimum positive integer which satisfies $\lambda_{D i}^{p_{D i}}=1$. Let the generalized eigenvector corresponding the eigenvalue $\lambda_{D i}$ be $v_{D i}$ and its depth be $q_{D i}$. For the vector $v_{D i}$, the depth $q_{D i}$ is defined by the positive number which satisfies $v_{D i} \in \operatorname{Ker}\left(\lambda_{D i} I-A_{D}\right)^{q_{D i}}$ and $v_{D i} \notin \operatorname{Ker}\left(\lambda_{D i} I-A_{D}\right)^{q_{D i}-1}$. Then the period of $i$ th eigenvector is given by $2^{g_{D i}} p_{D i}$ where $g_{D i}$ denotes a minimum integer satisfying $q_{D i} \leq 2^{g_{D i}}$. In a similar manner, the period of $j$ th eigenvector of $A_{R}$ is also given by $2^{g_{R j}} p_{R j}$.

Now we compute the period $p_{i j}$ for an eigen-connection $W_{i j}=v_{D i} v_{R j}{ }^{T}$ as

$$
p_{i j}=\operatorname{LCM}\left(2^{g_{D i}} p_{D i}, 2^{g_{R j}} p_{R j}\right) .
$$

Therefore the period $P$ for a connection $X$ is given by

$$
\operatorname{LCM}(P), P=\left\{p_{i j} \mid(i, j) \in C\right\}
$$

where $C$ represents a set of subscripts of eigen-connections occupied by $X$ given by $C=\left\{(i, j) \mid a_{i j} \neq 0, X=\right.$ $\left.\sum_{i=1}^{n} \sum_{j=1}^{n} a_{i j} W_{i j}\right\}$.

When a system has duplicate eigenvalues and the deeper eigen-connection space is excited, the period is doubled, quadrupled, etc. Also when a $m$-degree primitive polynomial is included in the characteristic polynomial, the maximum period $2^{m}-1$ is observed.

\section{E. Steady Connection}

In this section, a set of steady connections is derived.

When a pair of eigenvalues $\beta$ and $\gamma$ satisfies $\beta \times \gamma=1$, they are defined as contrary eigenvalues. The system (9) has a steady connection except $X=0$, if and only if $A_{D}$ and $A_{R}$ have a pair of contrary eigenvalues. Let $i$ th eigenvalues of $A_{D}$ be $\beta_{i}$ and its contrary eigenvalue of $A_{R}$ be $\gamma_{i}$. $A_{D}$ has other conjugate eigenvalues $\beta_{i}^{2}, \beta_{i}^{4}, \ldots, \beta_{i}^{2^{d-1}}$ and $A_{R}$ has also other conjugate eigenvalues $\gamma_{i}^{2}, \gamma_{i}^{4}, \ldots, \gamma_{i}^{2^{d-1}}$. Then each of them makes a pair of contrary eigenvalues, i. e., $\beta_{i}^{2^{j}} \times \gamma_{i}^{2^{j}}=1$ for $0 \leq j \leq d-1$. Let the corresponding eigenvectors be

$$
\begin{aligned}
V_{D i} & =\left[v_{D i 0}, v_{D i 1}, \ldots, v_{D i d-1}\right] \\
V_{R i} & =\left[v_{R i 0}, v_{R i 1}, \ldots, v_{R i d-1}\right]
\end{aligned}
$$

where $v_{D i j}$ denotes the eigenvector for the eigenvalue $\beta_{i}^{2^{j}}$ and $v_{R i j}$ denotes the eigenvector for the eigenvalue $\gamma_{i}^{2^{j}}$, i. e., $v_{D i j} \in \operatorname{Ker}\left(\beta_{i}^{2^{j}} I-A_{D}\right)$ and $v_{R i j} \in \operatorname{Ker}\left(\gamma_{i}^{2^{j}} I-A_{R}\right)$. Under these preparations, we have a following theorem.

Lemma 1: A set of steady connections is given by

$$
\Omega_{1}=\left\{X \mid X=V_{D i} V_{R i}{ }^{T}, i \in C\right\}
$$

where $C=\left\{i \mid \beta_{i} \gamma_{i}=1\right\}$.

Proof: The definitions of $V_{D}$ and $V_{R}$ yield

$$
\begin{aligned}
& A_{D} V_{D}=V_{D} \operatorname{diag}\left(\beta, \beta^{2}, \ldots, \beta^{2^{d-1}}\right) \\
& A_{R} V_{R}=V_{R} \operatorname{diag}\left(\gamma, \gamma^{2}, \ldots, \gamma^{2^{d-1}}\right)
\end{aligned}
$$


Note that each of eigenvalues is contrary, i. e., $\beta^{2^{j}} \gamma^{2^{j}}=1$ for $0 \leq j \leq d-1$. Then we compute

$$
\begin{aligned}
A_{D} V_{D} V_{R}^{T} A_{R}^{T} & =V_{D} \operatorname{diag}\left(\beta \gamma, \beta^{2} \gamma^{2}, \ldots, \beta^{2^{d-1}} \gamma^{2^{d-1}}\right) V_{R}^{T} \\
& =V_{D} V_{R}^{T}
\end{aligned}
$$

Therefore, the connection $X=V_{D} V_{R}^{T}$ is the steady connection which holds $X=A_{D} X A_{R}^{T}$. The necessity of the condition is trivial.

The system (9) has a vanishing connection which converges on the origin $X=0$ within finite steps if and only if $A_{D}$ or $A_{R}$ has eigenvalues 0 .

Lemma 2: A set of the vanishing connections is given by

$$
\begin{aligned}
\Omega_{0}= & \left\{X \mid X=v_{D} a_{R}^{T}+a_{D} v_{R}^{T}, v_{D} \in \operatorname{Ker} A_{D}{ }^{m_{D}},\right. \\
& \left.v_{R} \in \operatorname{Ker} A_{R}^{m_{R}}, \forall a_{D}, a_{R} \in G F(2)^{n}\right\} .
\end{aligned}
$$

The connection converges on 0 within $\max \left(m_{D}, m_{R}\right)$ steps, where $m_{D}$ denotes a minimum integer satisfying $\operatorname{rank} A_{D}{ }^{m_{D}}=\operatorname{rank} A_{D}{ }^{n}$. Also $m_{R}$ is defined for $A_{R}$ in a similar manner.

Proof: If either $A_{R}$ or $A_{D}$ has an eigenvalue 0 , a non-zero eigenvector $v_{D} \in \operatorname{Ker} A_{D} m_{D}$ or $v_{R} \in \operatorname{Ker} A_{R} m_{R}$ exists. In a case of $m_{D} \geq m_{R}$, we compute the connection in (44) after $m_{D}$ steps so that $A_{D}{ }^{m_{D}} X\left(A_{R}^{T}\right)^{m_{D}}=$ $A_{D}{ }^{m_{D}}\left(v_{D} a_{R}^{T}+a_{D} v_{R}^{T}\right)\left(A_{R}^{T}\right)^{m_{D}}=0$ because $A_{D}{ }^{m_{D}} v_{D}=$ 0 and $A_{R}^{m_{D}} v_{R}=A_{R}^{m_{D}-m_{R}} \times A_{R}^{m_{R}} v_{R}=0$. The case of $m_{D}<m_{R}$ is also computed in similar manner.

Thus, a set of connections which converge on a steady connection within finite steps is given by $\Omega_{0} \oplus \Omega_{1}$

\section{Control of Connection Systems}

The eigenvalues of the connection systems (9) can be designed using external event inputs $u, u_{D}$, and $u_{R}$. On (9), when we set connection feedback

$$
u=f_{D}^{T} X f_{R}, u_{D}=X^{T} f_{D}, u_{R}=X f_{R}
$$

then the following dynamics of the connection is obtained.

$$
X(t+1)=\left(A_{D}+b_{D} f_{D}^{T}\right) X(t)\left(A_{R}+b_{R} f_{R}^{T}\right)^{T}
$$

Any eigenvalues can be designed if and only if both $\left(A_{D}, b_{D}\right)$ and $\left(A_{R}, b_{R}\right)$ are controllable.

\section{Decentralized Implementation}

Let the connection matrix be $X=\left[x_{D 1}, \ldots, x_{D n}\right]=$ $\left[x_{R 1}, \ldots, x_{R n}\right]^{T}$. Each subsystem consists of $\left\{x_{D i}, x_{R i}\right.$, $\left.A_{D}, A_{R}\right\}$ where $x_{D i}, x_{R i} \in G F(2)^{n}$ and $A_{D}, A_{R} \in$ $G F(2)^{n \times n} \cdot x_{D i}$ and $x_{R i}$ represent local donator and receptor connections of $i$ th subsystems, respectively. Usually, the subsystems communicate signals each other depending on their donator and receptor connections $x_{D i}, x_{R i}$. The matrices $A_{D}$ and $A_{R}$ are homogeneous among subsystems. If an event occurs, the update procedure of connections is carried out as follows:

1. Update donator connections by $x_{D i} \leftarrow A_{D} x_{D i}$ for each subsystems.

2. Update receptor connection $x_{R j}$ to be consistent to $x_{D i}$ for $j \neq i$.
3. Update receptor connections by $x_{R i}{ }^{T} \leftarrow x_{R i}{ }^{T} A_{R}{ }^{T}$ for each subsystems.

4. Update donator connection $x_{D j}$ to be consistent to $x_{R j}$ for $j \neq i$.

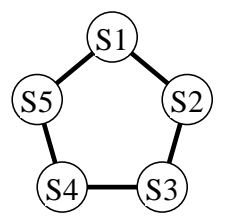

(a) Ring network

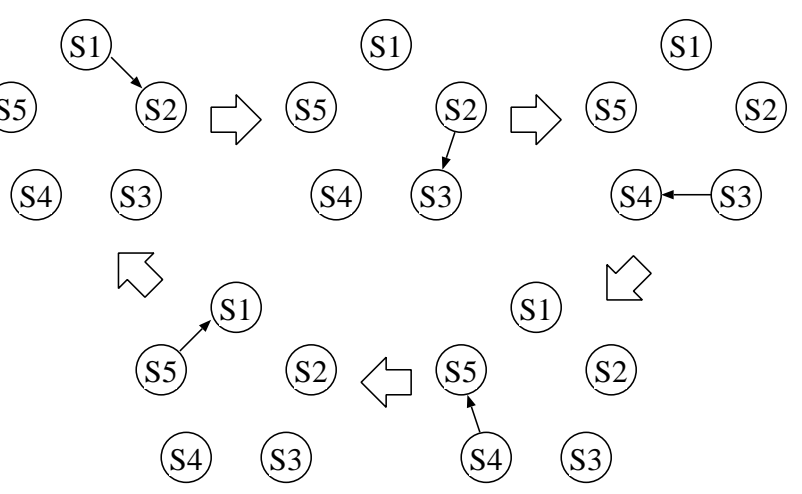

(b) Token passing sequence

Fig. 5. Token passing protocol

\section{Illustrative Examples}

\section{A. Token Passing Protocol on Ring Network}

Token Passing Protocol is a communication mechanism for multi-node network that realizes time critical communications. To avoid collisions, the node passes a token from one to another, and it has a right to talk on the network only if it holds the token.

Consider a ring network with five nodes as shown in Fig. 5. First, the token is passed from the node $S_{1}$ to the node $S_{2}$, then it is passed from the node $S_{2}$ to the node $S_{3}$. Namely, it moves cyclically as $S_{1} \rightarrow S_{2} \rightarrow S_{3} \rightarrow S_{4} \rightarrow$ $S_{5} \rightarrow S_{1}$.

The delivery of token is modeled as an autonomous system with informational connection: $X(t+1)=A_{D} X(t) A_{R}^{T}$ where

$$
\begin{aligned}
& \left\{A_{D}, A_{R}, X(0)\right\}= \\
& \left\{\left[\begin{array}{lllll}
0 & 0 & 0 & 0 & 1 \\
1 & 0 & 0 & 0 & 0 \\
0 & 1 & 0 & 0 & 0 \\
0 & 0 & 1 & 0 & 0 \\
0 & 0 & 0 & 1 & 0
\end{array}\right],\left[\begin{array}{lllll}
0 & 0 & 0 & 0 & 1 \\
1 & 0 & 0 & 0 & 0 \\
0 & 1 & 0 & 0 & 0 \\
0 & 0 & 1 & 0 & 0 \\
0 & 0 & 0 & 1 & 0
\end{array}\right],\left[\begin{array}{lllll}
0 & 0 & 0 & 0 & 0 \\
1 & 0 & 0 & 0 & 0 \\
0 & 0 & 0 & 0 & 0 \\
0 & 0 & 0 & 0 & 0 \\
0 & 0 & 0 & 0 & 0
\end{array}\right]\right\}
\end{aligned}
$$

The characteristic polynomials of $A_{D}$ and $A_{R}$ are computed as

$$
\begin{aligned}
& \psi_{D}(\lambda)=\psi_{R}(\lambda)=\lambda^{5}+1 \\
& \quad=(\lambda+1)\left(\lambda^{4}+\lambda^{3}+\lambda^{2}+\lambda+1\right) \\
& \quad=(\lambda+1)\left(\lambda+\alpha^{3}\right)\left(\lambda+\alpha^{6}\right)\left(\lambda+\alpha^{9}\right)\left(\lambda+\alpha^{12}\right)
\end{aligned}
$$


where $\alpha \in G F\left(2^{4}\right)$ is a root of 4 th degree irreducible polynomial $\lambda^{4}+\lambda+1=0$. From (48) the Donator eigenvalues $\lambda_{D}$ and the Receptor eigenvalues $\lambda_{R}$ are $\lambda_{D}=\lambda_{R}=$ $\left\{1, \alpha^{3}, \alpha^{6}, \alpha^{9}, \alpha^{12}\right\}$. Then eigenvalues of the system are calculated as $\left\{1, \alpha^{3}, \alpha^{6}, \alpha^{9}, \alpha^{12}\right\}$ by $(21)$ for $1 \leq i \leq 5$, $1 \leq j \leq 5$. The system has five fivefold eigenvalues. Fig. 6 shows GF-plane of the system. It is observed that the period of four eigenvalues $\left\{\alpha^{3}, \alpha^{6}, \alpha^{9}, \alpha^{12}\right\}$ is five.

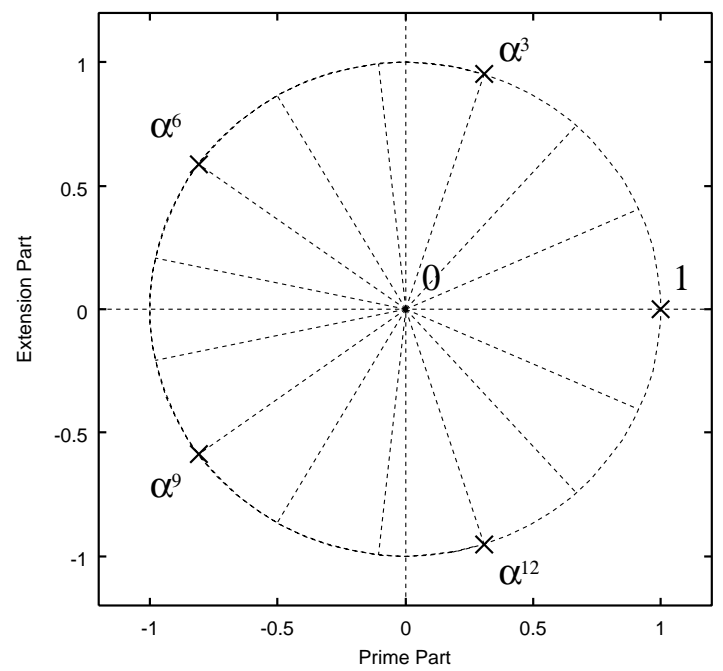

Fig. 6. Pole plot of token passing model

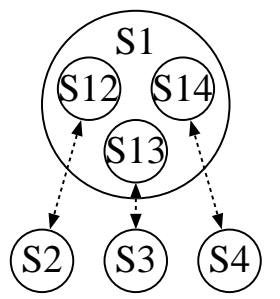

Fig. 7. Asynchronous 1-scheduler model

\section{B. Asynchronous 1-Scheduler}

The proposed modeling method is applied to an asynchronous 1-scheduler for simple parallel computers. There is a scheduler process that divides a job into multiple parts and dispatches them to the other processes. Let $S_{1}$ be the scheduler process and $S_{2}, S_{3}, S_{4}, \ldots$ be the other subprocesses. A sequence of a request $S_{1} \rightarrow S_{i}$ and an acknowledgement $S_{1} \leftarrow S_{i}$ is asynchronously realized.

Assume that only three sub-precesses $S_{2}, S_{3}, S_{4}$ exist. The scheduler process $S_{1}$ is divided into three parts according to the condition of the communication as shown in Fig. 7. Then we have the connection system: $X(t+1)=$ $A_{D} X(t) A_{R}^{T}+B_{D} \operatorname{diag}\left(u_{1}, u_{2}, u_{3}\right) B_{R}^{T}$ where

$$
\left\{A_{D}, A_{R}, B_{D}, B_{R}\right\}=
$$

$$
\left\{\left[\begin{array}{llllll}
0 & 0 & 0 & 1 & 0 & 0 \\
0 & 0 & 0 & 0 & 1 & 0 \\
0 & 0 & 0 & 0 & 0 & 1 \\
0 & 0 & 0 & 0 & 0 & 0 \\
0 & 0 & 0 & 0 & 0 & 0 \\
0 & 0 & 0 & 0 & 0 & 0
\end{array}\right],\left[\begin{array}{llllll}
0 & 0 & 0 & 0 & 0 & 0 \\
0 & 0 & 0 & 0 & 0 & 0 \\
0 & 0 & 0 & 0 & 0 & 0 \\
1 & 0 & 0 & 0 & 0 & 0 \\
0 & 1 & 0 & 0 & 0 & 0 \\
0 & 0 & 1 & 0 & 0 & 0
\end{array}\right],\left[\begin{array}{lll}
0 & 0 & 0 \\
0 & 0 & 0 \\
0 & 0 & 0 \\
1 & 0 & 0 \\
0 & 1 & 0 \\
0 & 0 & 1
\end{array}\right],\left[\begin{array}{lll}
1 & 0 & 0 \\
0 & 1 & 0 \\
0 & 0 & 1 \\
0 & 0 & 0 \\
0 & 0 & 0 \\
0 & 0 & 0
\end{array}\right]\right\}
$$

where event input $u_{i}$ denotes a request flag from the scheduler to $(i+1)$ th sub-process.

Since the characteristic equations of the Donator matrix $A_{D}$ and the Receptor matrix $A_{R}$ are $\psi_{D}(\lambda)=\psi_{R}(\lambda)=$ $\lambda^{6}=0$, the system has sixfold poles. The corresponding eigenvectors are calculated as $v_{D 1}=[1,0,0,0,0,0]^{T}$, $v_{D 2}=[0,0,0,1,0,0]^{T}, v_{D 3}=[0,1,0,0,0,0]^{T}, v_{D 4}=$ $[0,0,0,0,1,0]^{T}$,

$v_{D 5}=[0,0,1,0,0,0]^{T}, v_{D 6}=[0,0,0,0,0,1]^{T}, v_{R 1}=$ $[0,0,0,1,0,0]^{T}, \quad v_{R 2}=[1,0,0,0,0,0]^{T}$, $v_{R 3}=[0,0,0,0,1,0]^{T}, \quad v_{R 4}=[0,1,0,0,0,0]^{T}, v_{R 5}=$ $[0,0,0,0,0,1]^{T}, v_{R 6}=[0,0,1,0,0,0]^{T}$. Thus the system has 36 eigen-connections given by $(20)$. From $B_{D}$ and $B_{R}$, the eigen-connections excited by the external inputs $\left\{u_{1}, u_{2}, u_{3}\right\}$ are $\left\{W_{22}, W_{44}, W_{66}\right\}$. These eigen-connections represent the dispatches of the jobs from the scheduler to the sub-processes.

The eigen-connection $W_{22}$ is composed of two eigenvectors $v_{D 2}$ and $v_{R 2}$. Since the depth of these vectors is 2 , dynamics of the sub-space is governed by $W_{11}=A_{D} W_{22} A_{R}^{T}$ instead of (21). The sub-spaces $W_{44}$ and $W_{66}$ are governed by $W_{33}=A_{D} W_{44} A_{R}^{T}$ and $W_{55}=A_{D} W_{66} A_{R}^{T}$ as well. The eigen-connections $\left\{W_{11}, W_{33}, W_{55}\right\}$ represent the acknowledgements from sub-processes.

Then, the sub-spaces $\left\{W_{11}, W_{33}, W_{55}\right\}$ are governed by (21), namely $0=A_{D} W_{11} A_{R}^{T}, \quad 0=A_{D} W_{33} A_{R}^{T}, 0=$ $A_{D} W_{55} A_{R}^{T}$. They correspond to ends of the jobs.

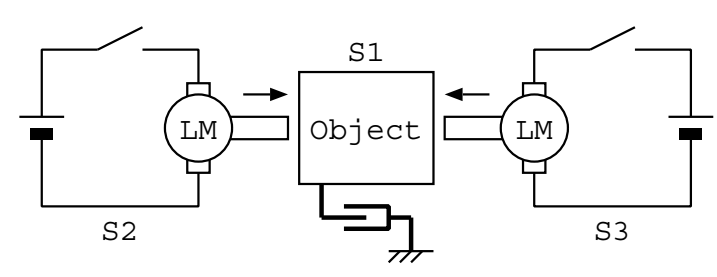

Fig. 8. Decentralized acutuation system

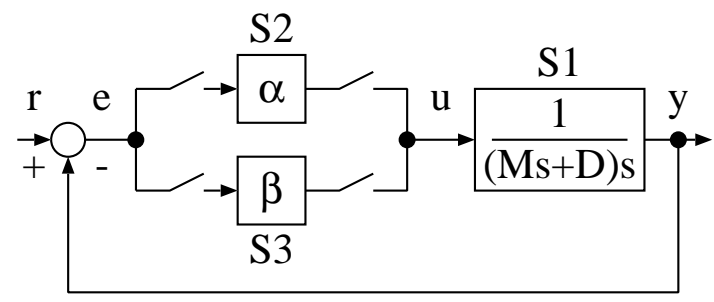

Fig. 9. Variable structure system with sliding mode 


\section{Variable Structure System}

Fig. 8 shows an illustrative example of a decentralized positioning system with a object and two one-way linear actuators. The system is regarded as a variable structure system. Fig. 9 shows a block diagram of the system where $M$ is inertia of object, $D$ is dumping factor, $r$ is reference position, $y$ is position of object, $e$ is error signal, $u$ is force imposed on object, and $\alpha$ and $\beta$ are gains for actuator \#1 and \#2, respectively.

Since the system has two actuators, we consider the dynamic connection system:

$$
\begin{aligned}
& \left\{A_{D}, A_{R}, b_{D}, b_{R}, X(0)\right\} \\
& \quad=\left\{\left[\begin{array}{lll}
1 & 0 & 0 \\
0 & 1 & 0 \\
0 & 0 & 1
\end{array}\right],\left[\begin{array}{lll}
1 & 0 & 0 \\
0 & 1 & 0 \\
0 & 0 & 1
\end{array}\right],\left[\begin{array}{l}
0 \\
1 \\
1
\end{array}\right],\left[\begin{array}{l}
0 \\
1 \\
1
\end{array}\right],\left[\begin{array}{lll}
0 & 1 & 0 \\
1 & 0 & 0 \\
0 & 0 & 0
\end{array}\right]\right\} .
\end{aligned}
$$

The external event inputs are given by the connection feedback (45) and $f_{D}=f_{R}=[0,1,1]^{T}$. The event inputs are activated when $\sigma(t) \times \sigma\left(t-T_{s}\right)<0$ where $\sigma(t)$ is defined by $\sigma(t)=c e(t)+\dot{e}(t)$ and $T_{s}$ is the control period. $\sigma(t)=0$ denotes sliding surface. The connection feedback yields controlled system matrices:

$$
A_{D}+b_{D} f_{D}^{T}=A_{R}+b_{R} f_{R}^{T}=\left[\begin{array}{lll}
1 & 0 & 0 \\
0 & 0 & 1 \\
0 & 1 & 0
\end{array}\right] .
$$

The dynamic transition of the informational connection is shown in Fig. 10. The unit time in this representation is defined as the minimal interval of the events. The Donator eigenvalues and the Receptor eigenvalues are calculated as $\lambda_{D}=\lambda_{R}=\{1\}$. The system has triple poles. From (37), the period of the system becomes 2 because the depth of one of the eigenvectors is 2 . It corresponds to the fact that the system has two structures.

The system is implemented in decentralized subsystems connected via Fast Ethernet as shown in Fig. 11, where the personal computer PC1 emulates the object. The PC2 and PC3 are the controller $S_{1}$ and $S_{2}$, respectively. The protocol is implemented on the upper level over the UDP/IP. Fig. 12 shows the experimental result of the phase plane trajectory.

The proposed model gives an analytical system structure from the view point of the upper event layer. Also it gives an decentralized implementation model as shown in the section V.
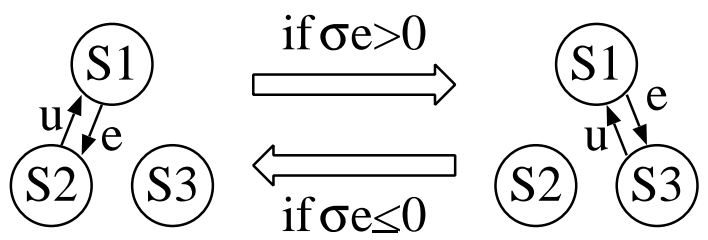

Fig. 10. Informational connection of VSS

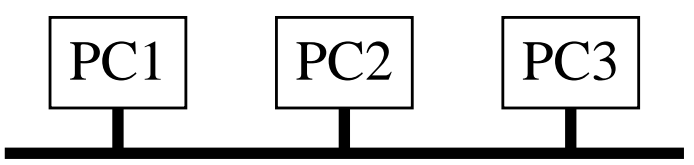

Fast Ethernet

Fig. 11. System configuration

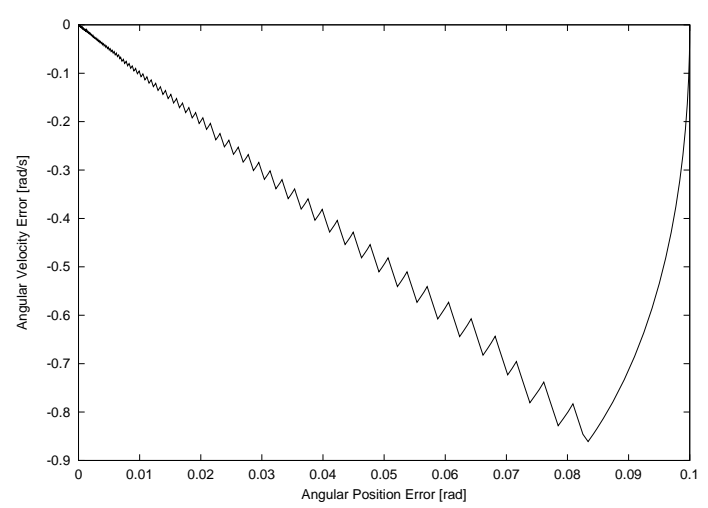

Fig. 12. Experimental results in phase plane

\section{CONCLUSIONS}

A design method of decentralized systems with informational connection is proposed. The dynamic transition of informational connection among decentralized systems is considered and the mathematical structures are discussed using a concept of eigenvalues and eigen-connections over the Galois field $G F(2)$. Examples of decentralized variable structure systems are shown. In an industry field, there are many engineering systems that have dynamic transition of informational connection. The mathematical model would be useful for analysis and synthesis of various informationally connected systems.

\section{REFERENCES}

[1] Y. Fujimoto, T. Seki, T. Yakoh, and K. Ohnishi, "Dynamic Model of Informationally Connected Systems," Trans. of IEE of Japan, vol. 120-D, no. 1, pp. 19-28, 2000. (in Japanese)

[2] T. Seki, Y. Fujimoto, T. Yakoh, and K. Ohnishi, "An Expression of Dynamical System Connection in Decentralized Autonomous Control," Trans. of IEE of Japan, vol. 120-D, no. 1, pp. 80-87, 2000. (in Japanese)

[3] K. Ueno, T. Yakoh, T. Murakami, and K. Ohnishi, "New Representation of Systems with Effect Variables," in Proc. IEEE Int. Conf. on Advanced Motion Control, pp. 216-221, 2000.

[4] S. Shin, K. Ikeda, H. Yuasa, and H. Fujita, "Autonomous Decentralized Systems," Asakura-Shoten, 1995. (in Japanese)

[5] R. Tanaka, S. Iwata, and S. Shin, "Structural Analysis of Faulttolerance for Homogeneous Systems," in Proc. IEEE Conf. on Decision and Control, pp. 1920-1921, 1996.

[6] K. Murota, "Eigensets and Power Products of a Bimatroid," Advances in Mathematics, vol. 80, pp. 78-91, 1990.

[7] N. Yamasaki and T. Matsui, "A Functionally Distributed Responsive Micro Controller for Distributed Real-time Processing," in Proc. IEEE/RSJ Int. Conf. on Intelligent Robots and Systems, pp. 793-798, 1997.

[8] T. Sekiguchi, T. Kagawa, H. Otto, H. Sasajima, J. H. Christensen, and A. Sugiura, IEC61499 User Workshop, JEMIMA, Yokohama, 1999.

[9] H. Imai, Coding Theory, IEICE, 1990. (in Japanese) 


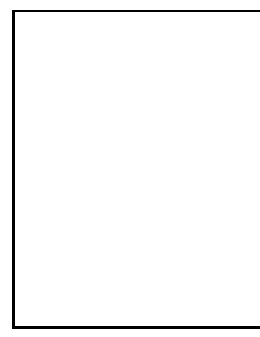

Yasutaka Fujimoto (S'93-M'98) received B.E., M.E., and Ph.D. degrees in electrical engineering from Yokohama National University, Japan, in 1993, 1995, and 1998, respectively. In 1998, he joined the Department of Electrical Engineering, Keio University. Since 1999, he has been with the Department of Electrical and Computer Engineering, Yokohama National University. His research interests include discrete systems, motion control, and robotics.

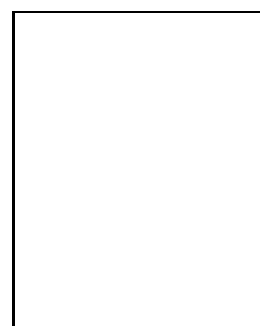

Takahiro Yakoh received the B.E. degree in instrumentation engineering, M.E. and Ph.D. degrees in computer science from Keio University, Japan, in 1989, 1991, 1995, respectively. Since 1998, he has been with the Department of System Design Engineering, Keio University, where he is currently an Assistant Professor. His main research area are communicating real-time systems, operating systems, computer networks, distributed systems, and robotics. He is a member of the IEEE Computer Society and Industrial Electronics Society.

Kouhei Ohnishi (S'78-M'80-F'01) received B.E., M.E., and Ph.D. degrees, all in electrical engineering from the University of Tokyo, Japan, in 1975, 1977, and 1980, respectively. Since 1980, he has been with Keio University. His research interests include mechatronics, motion control, and robotics. 